\title{
XVI.
}

\section{Ueber einen Fall von Kleinhirnschwund mit Degenerationen im verlängerten Marke und im Rückenmarke (wahrscheinlich in Folge von Alkoholismus).}

\author{
Von Prof. Dr. Schultze in Heidelberg.
}

So häufig bekanntlich atrophische Zustände im Grosshirn, Rückenmark und im peripherischen Nervensysteme sind, so selten werden sie im Kleinhirn angetroffen. Hitzig hat in seiner zusammenfassenden Darstellung der cerebellaren Atrophien in Ziemssen's Handbuch der speciellen Pathologie (I. Aufl.) nur über 11 Fälle aus der Literatur berichten können, wobei allerdings nur die hochgradigen Schrumpfungen des genannten Organes Berücksichtigung fanden. In meinem eigenen gleich mitzutheilenden Falle war nun die Affection keine so beträchtliche, wie in den meisten von Hitzig erwähnten, aber doch noch recht erheblich.

Ueber die Krankengeschichte dieses Falles vermag ich Folgendes zu berichten: Im Juli 1882 wurde mir ein 39jähriger Herr zur Behandlung überwiesen, welcher hauptsächlich drei Krankheitssymptome zeigte. Nebmlich 1) einen Diabetes insipidus bezw. eine Polydipsie mit Diabetes insipidus, der schon seit seiner Knabenzeit bestanden haben soll; 2) eine sehr erhebliche Gehstörung, welche darin bestand, dass der Kranke beim Gehen schwankte und taumelte, wie ein Betrunkener und deshalb auf der Strasse wenigstens stets geführt werden musste; 3) eine Sprachstörung, so dass die Worte ziemlich langsam und lallend, mit undeutlicher Articulation ausgesprocben wurden. Für eine typische "scandirende" Sprache war die Aufeinanderfolge der einzelnen. Silben und Worte nicht gleichförmig genug; eine Störung wie bei der Atrophie des Hypoglossuskernes (bei der amyotrophischen Bulbärparalyse) lag ebenfalls nicht vor. Zeichen von Zungenatrophie feblten; ebensowenig waren tabische Symptome vorhanden; von dem Romberg'schen Symptome liess sich nichts wahrnebmen. Schwindelgefübl war nicht selten vorhanden, hie und da einmal Kopfweh und Erbrechen. Die Intelligenz war nicht schwächer geworden. In Bezug auf die Aetiologie liess sich durch die Bemähungen des Hausarztes, Herrn Dr. Nebel, eruiren, 
dass Lues niemals bestanden hat, dass aber der Patient Jabre lang eine grosse Menge Pfälzer Weines, 6-10 Flaschen täglich, zu sich nahm und daneben noch grosse Quantitäten Wasser brauchte. In den letzten Jahren wurde allerdings dieser Abusus vini nicht mehr geïbt, aber noch stetig reichlich Wasser getrunken. Der Harn war demgemäss vou geringen specifischem Gewicht, übrigens ohne Aibumin und Zucker. Eine mehrwöchentliche galvanische Kur blieb obne jeden Erfolg. Da die Krankheit langsan weitere Fortschritte machte, wurde etwa $\frac{5}{4}$ Jahre später auch Herr. Prof. Erb consultirt, welchem ich die folgenden Notizen über den Kranken verdanke, den ich nach dem Jahre 1882 nicht wieder zu seben Gelegenheit hatte. Anamnestisch wurde eruirt, dass der Diabetes insipidus schon seit dem 12. Lebensjahre bestanden haben soll; innerbalb der letzten 2 Jabre seien öfters Ziehen und Parästhesien in den Beiren anfgetreten, ausserdem "Gürtelgefühi" und eine gewisse Blasenschwäche. Auch die Hände sind in letzter Zeit unsicher geworden.

Erb constatirte bei der Untersuchung Folgendes: Leichter Nystagmus; Pupillen gut; Sprache undeutlich, nicht scandirend.

In den Armen Spur von Intentionszittern; Gang unsicher und nur bei Führung mëglich. Zehenstand gut. Romberg'sches Symptom fehlt. Patellarreflexe sebr lebhaft; kein Fussklonus. Sensibilität gut (wie auch früher). Innere Organe gesund; keine Impotenz.

Nach einem weiteren Vierteljabre war wiederum Verschlimmerung eingetreten; es bestand etwas Fieber, grössere Schwäche; deutlicheres Zittern; der Durst und die Urinmenge waren geringer.

Nach der Mittheilung des Hausarztes nahm dann später die Sprachstörung immer mebr zu, so dass der Kranke zuletzt völlig unverständlich wurde; das Kopfweh trat viel bäufiger ein; die Gehschwäche steigerte sich zur Läbmung; schliesslich stellten sich erhebliche Anfälle von nervöser Dyspnoe ein (Dr. Stadelmann); und unter zunehmender Respirationslähmung trat am 30. Mai 1886 der Tod ein.

Epileptische Anfälle fehlten während der ganzen Dauer der Affection. Angesiehts der seinerzeit sehr charakteristischen Gehstörung hatte ich die Diagnose auf ein cerebellares Leiden gestellt, ohne dass ich aber den Diabetes insipidus und die Sprachstörung auf eine bestimmte Läsion zurüekzuführen wagte; ein Gliom des Cerebellum schloss ich aus, sowohl wegen der nur wenig hervortretenden Kopfschmerzen, als auch wegen der allzulangsamen Entwickelung des Leidens und der fehlenden Sehstörungen. Welcher Art der zu Grunde liegende Prozess sei, liess ich dahingestellt; Erb, welcher den Kranken in vorgeschrittenerem Stadium seiner Affection sah, nahm eine cerebrospinale Sklerose als das Wahrscheinlichste an.

Die am 31. Mai 1886 von Hern Dr. Fleiner vorgenommene Section ergab nur in erster Linie eine ausgesprochene A trophie und Sklerose des Kleinhirns und der Medulla oblongata. Am Grosshirn war keine deutliche Veränderung makroskopisch sichtbar; ebensowenig am Rückenmarke. Die basalen Arterien des Gehirnes zeigten eine ungleichmässige Verdickung 
jhrer Wand, eine Endarteriitis obliterans. Anzeichen von Sypbilis fanden sich nirgends. An den inneren Organen wurden ausser einer Hypertrophie und Dilatation der Harnblase und einer Hydronephrose besonders der linken Seite nichts Abnormes constatirt.

Das Kleinbirn mit der Med. obl. und die Medulla spinalis wurden dann in gewöhnlicher Weise in Müller'scher Flüssigkeit und Alkohol gehärtet, nur dass die Härtung nach dem Vorschlage des jüngeren Virchow im Dunkeln vorgenommen wurde. Die Niederschläge, welche sonst, bei der gewöhnlichen Behandlung im Lichte, nach der Ueberführung in Alkohol ohne vorherige Auswässerung zu entstehen pflegen, blieben allerdings wie bei andern ebenso behandelten Präparaten aus; ich konnte indessen einen Unterschied in der Tinctionsfähigkeit der von mir angewendeten Färbemittel weder in diesen noch in den andern analog behandelten Präparaten im Vergleich $z u$ den unter der Einwirkung des Lichtes gehaltenen entdecken. Sowobl die Färbungen mit Boraxcarmin und Alauncarmin, als die Weigert'sche Tinction gelangen bei beiden Arten von Präparaten unter sonst gleichen Umständen głeich gut. Was zuerst das Kleinhirn selbst angeht, so waren die Dimensionen desselben gegenäber anderen mit demselben verglichenen Präparaten erwachsener Männer allseitig erheblich verringert.

Die grösste Ausdehnung des Wurmes von vorn nach binten in der Medianebene betrug $3 \mathrm{~cm}$ (an damit verglichenen Präparaten Erwachsener

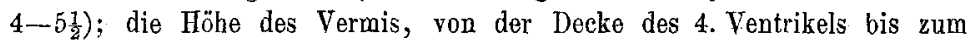
gegenüberliegenden höchsten Punkte maass $2 \frac{1}{2} \cdot \mathrm{cm}$, an Controlpräparaten $3-4 \mathrm{~cm}$.

Das Kleinhirn selbst hatte eine grösste Breite von nur $9 \mathrm{~cm}$, gegenüber den von mir verglichenen Normalmaassen von $11 \frac{1}{2}-12 \frac{1}{2} \mathrm{~cm}$ (bei männlichen Erwachsenen), eine grösste Länge (in der Richtung von den Vierhügeln nach dem Rückenmarke zu) von $3 \frac{3}{4} \mathrm{~cm}$ (Controlmaasse an Gesunden : 5 5 - -7 ) und maass endlich in der maximalen Höhenausdebnung (von der Basis des Gebirns bis nach oben) $5 \mathrm{~cm}$, gegenüber etwa $6 \mathrm{~cm}$ bei den Normalpräparaten.

Besonders stark erschien die Marksubstanz verschmälert; aber auch die Dicke der Rinde ist an vielen Stellen schon für die Betrachtung mit dem blossen Auge unzweifelhaft vermindert. Eine abnorme kleinhöckrige Form der Rindenoberfläche lässt sich nicht wahrnehmen; der Schwund ist ein mebr gleichnässiger; an Schnitten, welche in verschiedenen Riehtungen durch das Kleinhirn gehen, bemerkt man indess eine umschriebene Verfärbung einzelner Theile, so dass eine heerdförmige Erkrankung an denselben für das Auge deutlich wird. Es bildet indess diese Erscheinung nur ein ansnahmsweises Verhalten. In der Rindensubstanz des Wurmes zeigen sich ganz kleine Fleckchen von andersartiger Beschaffenheit als die Umgebung. Die Pia mater ist wohl etwas dicker als sonst, aber keineswegs in auffallendem Maasse.

Die Med. oblongata ist im Allgemeinen ebenfalls kleiner als normal, obne dass aber einzelne Theile derselben besonders stark atrophiseb erschienen; eine fleckweise auftretende Erkrankung derselben ist nicht nachweis- 


\section{4}

bar. - Die Arteria basilaris sowie die beiden Vertebrales sind erheblich verändert. Während die erstere mehr gleichförmig, aber nur in mässigem Grade verdickt ist, zeigt die linke Vertebralis sowohl dicht an ihrer Einmündungsstelle in die Basilaris als etwa $2 \mathrm{~cm}$ unterhalb derselben eine hocbgradige Endarteriitis, so dass in der Näbe der Basilaris das Lumen des Gefässes nur noch von sehr geringfügiger Ausdehnung ist. Die rechte Vertebralis ist wieder mehr gleichmässig verdickt obne stärkere Einengung ihres Lumen an irgend einer Stelle.

Am Rückenmarke zeigt sich eine deutliche Verfärbung in der Gegend der Pyramidenbahnen, welche in der Halsanschwellung nur schwach ausgebildet ist und nach der Oblongata hin sich völlig verliert, während sie im ganzen Dorsaltheil sehr ausgeprägt ist, um in der Lendenanschwellung wieder an Deutlichkeit einzubüssen.

Die genauere mikroskopische Untersuchung des Kleinhirns ergab zunächst nach der Anwendung der Weigert'schen Hämatoxylinmethode einen ganz erbeblichen Verlust an normalen Markscheiden, welcher innerhalb der. central gelegenen weissen Substanz am stärksten ausgeprägt war. In ungleichmäsșiger Weise zeigt sich die unmittelbar unter der grauen Substanz innerhalb der einzelnen Gyri gelegene Marksubstanz ergriffen; während manche Gyri an den angefertigten Querschnitten keinen wesentlichen Verlust an Markscheidensubstanz aufzuweisen hatten, lässt sich an anderen nur noch ein schwacher Rest derselben aufinden. Meistens zeigen sich dabei die am meisten central gelegenen Abschnitte der Marksubstanz am stärksten ergriffen ${ }^{1}$ ). Aber nur an den wenigen Stellen der Marksubstanz, welche sich schon bei makroskopischer Betrachtung als besonders stark verändert erwiesen, fehlt die Weigert'sche Reaction ganz; im Allgemeinen handelt es sich sonst um eine verschieden starke Rarefication und Verdünnung der Markscheiden, also anders wie bei der gewöbnlichen multiplen Sklerose, bei welcher die Plaques gewöbinlich eine scharfe Grenze gegen die noch normale Umgebung zeigen, weil die Markscheiden sehr rasch ihr Mark zu verlieren pflegen.

Neben diesem Minus von Markscheide und ebenso von Axencylindern ist đann eine erhebliche Anhäufung von Glia, besonders an den heerdförmig veränderten Stellen nachweisbar; nirgends ist aber eine Anhäufung ron Rundzellen, wie bei Granulationsgeschwülsten zu erblicken. Die Glia ist an manchen Stellen ziemlich kernreich, an anderen wieder kernarm. Dabei sind die Gefässe, und zwar besonders die Arterien, durchweg stark verândert; überall finden sich die Bilder einer Verdickung der Intima oder der Adventitia oder beider; die perivasculären Räume sind stellenweise mit Rundzellen in mässiger Menge angefüllt; die Gefässe selbst zeigen kaum stärkere Durehsetzungen mit Zellen.

1) Ganz ähnliche Veränderungen wie die hier geschilderten hat neuerdings Friedmann im Neurol. Centralblatt (1888. No. 4 u. 5) beschrieben und abgebildet; nur betrafen sie bei ihm das Mark der Grosshirnhemisphären. 
Was die grane Substan z betrifft, so ist die Rinde vor Allem an Purkinje'schen Zellen sehr verarmt; eine grosse Anzahl derselben ist verkleinert und atrophisch; ihre Fortsätze sind ebenfalls verändert, zum Theil völlig geschwunden, zum Theil sehr dünn. Die Axencylinderzüge in viel geringerer Zahl als normal vorbanden; dabei liegen in der Axencylinderschicht überall zahlreiche Haufen von verändertem Blutfarbstoff, in Gestalt von rostbraun gefärbten Pigmenthaufen, deren Form meist eine rundliche ist und deren Grösse oft derjenigen des Zellkörpers einer Purkinje'schen Zelle gleichkommt. In der Schicht dieser Zellen, sowie in den nächst anliegenden Abschnitten der Axencylinderschicht fehlen die Markscheiden durchaus nieht vollig; sie baben sich sogar besser und vollständiger gefärbt, als bei manchen normalen Controlpräparaten, zum sicheren Beweise dafür, dass die Technik der Weigert'schen Färbung eine fehlerfieie war, wie sich übrigens auch daraus erkennen liess, dass diejenigen Nervenfasern in der Marksubstanz, welche sich überhaupt färbten, eine sehr intensive Reaction zeigten. Ueber das normale Verbalten dieser Fasern innerbalb der Rindensubstanz des Kleinhirns ist meines Wissens überbaupt noch keine eingehende Untersuchung mitgetheilt worden. In dem untersuehten atrophischen Kleinhirn fanden sich an gewissen Stellen der Gyri, und zwar an denjenigen, welche von der Kuppe derselben am weitesten entfernt lagen, stets tangentiale Fasern in der Purkinje'schen Zellenschicht und noch weiter in die Axencylindersehicht hinein, stellenweise sogar bis über die Mitte derselben hinausreichend. In der Scheitelgegend der Gyri dagegen strahlen die aus der Körnerschicht bezw. der darunter gelegenen Marksubstanz kommenden Fasern in immer senkrechter werdender Richtung, also parallel mit den Axencylindern der Purkinje'schen Zellen in die Rindensubstanz bis etwa zur Mitte derselben deutlich erkennbar hinein.

Innerbalb der Körnerschicht selbst, die im Allgemeinen am wenigsten gelitten hat, sind die Nervenfasern bezw. die Markseheiden keineswegs völlig geschwunden, aber doch seltener zu finden als an Normalpräparaten. Die Anhäufung von Blutfarbstoff findet sich auch in der Marksubstanz, ganz ähnlich, wie max das etwa bei Erweichungsheerden alten Datums in den Grosshirnhemisphären zu sehen bekommt.

Die Pia mater ist verdickt, aber ohne cireumscripte Anhäufungen von Rundzellen, ihre Gefässe lange nicht so stark verăndert, als diejenigen der Kleinhirnsubstanz selbst.

Alle geschilderten Veränderungen finden sich sowohl am Wurm, wie an den beiden Hemisphären ausgeprägt; an ersteren durchweg sehr stark, in den letzteren in verschiedener Intensität.

Eine eigentbümliche Veränderung fand sich bei der Untersuchung: des Corpus dentatum. Dasselbe ist vor Allem stark atrophisch; ein grosser Theil seiner Zellen ist völlig verschwunden, ein anderer Theil klein und stark pigmentirt; die Markscheiden sehr spärlich und an manchen Stellen ganz versehwunden. Dann aber ist an den nach der Weigert'schen Bämatoxylinmethode gefärbten Präparaten sehr auffällig das Auftreten massen- 
hafter, tief schwarz gefärbter, in Windungen und Krümmungen verlaufender Züge, welche bei oberflächlicher Betrachtung den Eindruck machen, als ob es sich um eine grosse Menge dicht aneinander gedrängter Capillaren und stärkerer Gefässe handle, die strotzend mit schwarz gefärbten Blutkörpern gefüllt sind. Die genauere Untersuchung und der Vergleich mit Carminpräparaten ergiebt aber bald, dass es sich nicht etwa um Blutkörper, sondern um unregelmässig geformte, körnig oder schollig vertbeilte Kalkmassen handelt, welche der Wand der Capillaren anliegen oder an grösseren Arterien in die Adventitia eingelagert sind, während die Intima freibleibt. Besonders an Querschnitten kleiner Arterien kann man diese Einlagerung in Form von ringförmigen Figuren auf das Dentlichste wahrnehmen. An vielen Capillaren ist auch eine weniger ausgebreitete Auflagerung bezw. Einlagerung von Kalk zu sehen, welcher dann mehr in der Form von einzelnen Körnchen auftritt. Sonderbarerweise finden sich aber nur diejenigen Blutgefässe in der angegebenen Weise verändert, welche innerhalb der Ganglienmassen des Corpus dentatum liegen und den vielfachen Windungen dieser Masse folgen, so dass ein Theil dieser Windungen - denn nicht alle sind betroffen - auf das Deutlichste durch die Kalkablagerung von dem übrigen Gewebe abgehoben wird. In manchen Ganglienzellen lassen sich ebenfalls tief schwarz gefärbte Kalkkörnchen nachweisen; in den übrigen Theilen des Kleinhirns, also ausserhalb des Corpus dentatum, fand sich an den untersuchten Abschnitten nichts dergleichen vor 1$)$. - Soweit die Veränderungen am Kleinhirn.

Selbstverständlich musste sich nun die Aufmerksamkeit weiterhin besonders auf etwaige secundäre Degenerationen richten, die durch den Schwund des Kleinhirns bedingt sein konnten. Indessen war ausser demselben nocb eine Entartung der Pyramidenbahnen und der Kernregionen des Vagus und Hypoglossus vorhanden, die sich nnmöglich auf eine primäre Degeneration des Kleinhirns beziehen liessen. Waren aber äberbaupt anderweitige, von der Cerebellaratrophie unabhängige Veränderungen in der Med. obl. und im Rückenmarke vorhanden, so liess sich bei Erkrankung anderer Bahnen kein Schluss auf ihre primäre oder secundäre Degeneration machen. Nur daraus, dass gewisse Bahnen, welche von einzelnen Autoren mit dem

1) Auch bei einem neuerdings untersuchten Falle von encephalitischen Erweichungsheerden bauptsächlich der Rindensubstanz, bei welchen die Untersuchung am frischen Präparate ausgedehnte Verkalkung der Rindenpyramidenzellen nachgewiesen hatte, zeigte sich nach Anwendung der Wं eigert'schen Methode eine intensiv schwarze Färbung der verkalkten Zellen, welche sich mit ihren zum guten Theil ebenfalls mit Kalk imprägnirten Fortsätzen auf das Deutlichste von dem gelblichen Grunde des sonstigen Gewebes abhoben. Es scheint somit die $\mathrm{W}$ eigert'sche Hämatoxylinfärbung ein gutes Reagens auf derartige Verkalkurgen zu sein, worüber noch weitere Untersucbungen Aufschluss zu schaffen haben werden. 
Cerebellum in Verbindung gebracht sind, und die nacb primärer Erkrankung desselben secundär degeneriren sollen, nicht degenerirt sind, liess sich eine Folgerung in dem Sinne ableiten, dass sie nicht secundär zu erkranken brauchen. Aber auch in dieser Richtung blieb zu berücksichtigen, dass der Kleinhirnschwund kein vollkommener und kein gleichmässiger ist, somit auch keine vollständigen. Entartungen zugeböriger Bahnen erwartet werden durften.

In erster Linie war nun an der Med. oblong. eine erbebliche A trophie beider Oliven wahrnehmbar, deren Ganglienzellen theilweise ganz geschwunden oder verkleinert waren, zum grösseren Theile indessen noch erbalten geblieben sind. Die in den Hilus der Oliven eintretenden Markscheiden ebenso wie die dieselben umgebenden Markschichten (das sogenannte Vliess) in ungleichmässiger Weise und in manchen Höhenabschnitten recht erheblich atrophirt und ihres Markes beraubt.

Im Einzelnen lässt sich in den verschiedenen Querschnittshöhen der Medull. obl. Folgendes constatiren:

In der Höbe der Pyramidenkreuzung selbst zeigen sich nur die Pyramiden bei Weigert'scher Färbung diffus etwas schwächer gefärbt als normal, sonst lässt sich etwas Abnormes nicht entdecken. In der Höhe des Vagusaustrittes sind die Pyramiden an Weigert'schen Präparaten ebenfalls etwas heller; die Olivenzwischenschicht dagegen normal gefärbt, dunkler als die Pyramiden. Die Oliven sind klein, ihre Platten dünn; um sie herum und in ihnen dunkel gefärbte Marksubstanz. Das Corpus restiforme zeigt sich intact; die anfsteigende Trigeminuswurzel desgleichen. Der Hypoglossuskern ist etwas schwächer gefärbt; ob auch der Vaguskern, lässt sich schwer beurtheilen, da derselbe auch unter normalen Umständen oft nur äusserst wenige Markfasern erkennen lässt. An Carmi npräparaten sind die Pyramiden vielleicht etwas gliareicher, in deutlicher Weise dagegen der Hypoglossuskern, vielleieht auch der Vagus; die Anzabl der Olivenganglienzellen nicht deutlich vermindert.

Weiter nach oben zu, aber noch unterhalb des inneren Acusticuskernes, sind die Pyramiden (makroskopisch an Schnittpräparaten betrachtet, die nach W eigert'scher Methode hergestellt sind) ebenso stark gefärbt, wie die Olivenzwischenschicht, nicht deutlich abnorm. In einem nach binten gelegenen Theile der letztgenannten Schieht allerdings etwas Entfärbung. Das Vliess der deutlich atrophischen Oliven zum Theil entfärbt, in ungleichmässiger Weise verdünnt; die den Hilus ausfüllenden Fasern zum Theil völlig entfärbt. Ausserdem ist in der binteren Hälfte der Raphe, ebenso wie in den angrenzenden Theilen der netzförmigen Substanz starke Entfärbung wahrnehmbar. Die Corp. restiformia in ihrer äusseren Hälfte intact; nach innen zu eine partielle Entfärbung, welche sich bis an die Olivenkörper selbst hinzieht. Es bildet diese entfärbte Stelle im Allgemeinen die Figur eines Halbmonds. Die nach aussen und nach den Oliven zu convexe Begrenzungslinie desselben ziebt etwa in der Richtung der bogenförmigen Fasern, welche von dem Corp. restiforme an die Oliven berangehen, bleibt aber stets von der Pia zwischen Corp. rest. und Olivengegend durch normal ge- 


\section{8}

färbte Faserzüge des Fibr. areuat, ext. getrennt. Der vordere Theil dieser Linie legt sich an den hinteren Theil des Vliesses der Oliven an; die inzere coneave Begrenzungslinie der halbmondförmigen Figur hält sich nicht sehr weit von der äusseren entfernt. - Die Hypoglossuskerne sind abnorm schwach gefärbt.

Die genauere mikroskopische Untersuchung der Hämatoxylinpräparate bestätigt natürlich die Ergebnisse der makroskopischen Betrachtung, zeigt aber noch weiterhin, dass auch in dem zwischen den Oliven gelegenen Theile der Raphe weniger markhaltige Fasern sich befinden. Ebenso sind die Querfasern, welche direct durch die Raphe hindurch von einer Olive zur anderen gehen, an Zahl bedeutend verringert; der Hilus ist stark rareficirt; ebenso sind verschiedene der büschelförmigen Markstrablungen in die einzelnen Windungen der Oliven ausserordentlich an Markscheidensubstanz verarmt. Die um die Convexitätsgrenze der Oliven herumgehenden Bogenfasern fehlen zum grossen Theil, ebenso die Verbindungsfasern zwischen den Oliven und dem Corpus restiforme, welche in den inneren Theil desselben einstrahlen und zum grossen Theil innerhalb des beschriebenen halbmondförmigen Heerdes sich befinden. Die Untersuchung an Carminpräparaten zeigt, dass an allen bezeichneten markarmen Partien die Axencylinder ebenfalls fehlen oder in abnorm geringer Zahl vorhanden sind. Die Hypoglossuskerne, die Vaguskerne, die netzförmige Substanz, zum Theile auch die Gegend zwischen Oliven und Corpus restif. sind abnorm kern- und gliareich; die vom Hypoglossuskern in die zugehörigen. Wurzeln austretenden Fasern erscheinen intact; die Stilling'schen Bündel sind etwas kernreicher. Die Gefässe der Med. obl., und zwar besonders die arterielien Stämmchen zum grossen Theil erheblich verdickt, an manchen Stellen stark mit Zellen durchsetzt und eingefasst.

In der Höhe des Eintrittes der Acusticuswurzeln verhalten sich die Pyramidenbabnen wie in den eben geschilderten Querschnitten. Die Olivenzwischenschicht ist in ihrem dorsalen Abschnitte etwas beller. Nur das àorsale Olivenblatt nach aussen und innen von Nervenmark umgeben; am ventralen Blatte und im Hijus fehlt die normale schwarze Färbung an Präparaten, die nach der We igert'sehen Methode gefärbt sind, völlig; an der Umbiegungsstelle beider Blätter ist das Marl wieder orkalten. Die Schleifensehicht ist abnorm hell gefärbt, die netzförmige Substanz ungleichmässig entfärbt. In der Region zwischen Oliven und Corp. restif. ist nur die an die Pia angrenzende Randzone in sehr schmaler Breite schwarz, die ganze innere Partie hell. Die äusseren zwei Drittel der Corp. restif. intact; im inneren Drittel in der Nähe der durchziehenden medianen Acusticusbündel eine deutliche Entfärbung. Die Trigeminuswurzel und der innere Acusticuskern von normalem Aussehen.

An Carminpräparaten ist die innerste Windung des dorsalen Olivenblattes beiderseits stark roth gefärbt und ausserordentlich kernreich; die Ganglienzellen sehr selten, aber auch in den übrigen Theilen der Oliven weniger häufig als normal, zum Theil klein, und pigmentirt. Auch die 
Raphe zum Theil tiefer gefärbt; im Corp. restiforme makroskopisch nichts Auffallendes. Die Acusticusfasern intact. An don verfärbten Partion, die sich bei der Weigert'schen Färbung als solche zeigen, grössere Mengen von Gliakernen; an den erheblich degenerirten Partien in den Oliven und um dieselben herum auch Schwund der Axencylinder. Die Gefässe auch hier, wie oben geschildert.

In der $\mathbf{H o ̈ h}$ e des Facialisaustrittes sind die Pyramiden etwas schwächer gefärbt, die Raphe und das Corp. trapezoides im Allgemeinen (an Präparaten nach Weigert'scher Methode) stark entfärbt. Auch die oberen Oliven sehr hell. Facialiskerne und Facialismuskeln normal. - Die oberfläehlichen Ponsfasern sind leidlich erhalten; dagegen die tieferen ebenso wie die Querfasern des Corp. trapez. stark rareficirt. Ausserdem erseheint auch ein Theil der vom Kleinhirn in den Pons einstrahlenden Fasern entfärbt, und zwar dorsalwärts gelegene. Innerbalb der oberen Olive auf der einen Seite (medianwärts von dem austretenden Facialisstamm, an der Grenze des Corp. trapez., und ventralwärts von dem Facialiskern) lässt sich starke Verminderung der Nervenfasern und auch der Ganglienzellen nachweisen. Zugleich zeigt sich hier dieselbe Verkalkung kleinerer Blutgefässe und Capillaren, wie wir sie im Corpus dentatum vorgefunden haben. Innerhalb der anderen oberen Olive sind die Veränderungen an den Fasern und Ganglienzellen weniger deutlich ausgeprägt; die Verkalkung der Gefässe feblt an den untersuchten Schnitten.

Weiter nach oben zu, in der oberen Hälfte des Pons und in der Höhe der unteren Vierhügel lässt sich eine ausgebreitete Degeneration der Querfasern in dem ganzen ventralen Theile des Pons nachweisen; auch die verticalen Faserbündel, mit Einschluss der Pyramidenbahnen, färben sich nicht so intensiv als normal. In dem Haubentheile des Querschnittes sind besonders die Bindearme bis zu ihrer Kreuzungsstelle hinauf ganz erheblich degenerirt, während die obere und untere Schleife sich zwar nicht völlig intact erweisen, aber doch sehr viele normal anssehende Fasern führen. Es bleibt indess auffallend, dass in der ganzen Med. oblong. durch die Reductionsflüssigkeiten, und zwar sowohl durch das von.Weigert angewendete Blutlaugensalz, wis das von $\mathrm{Pal}$ neuestens empfohlene hypermangansaure Kali mit nachfolgender Oxalsäure - Natr. sulfurosum-Behandlung, in Allgemeinen eine raschere Entfärbung der ganzen Querschnitte stattfindet, als bei Normalpräparaten. Die Kernregionen sind im Wesentlichen intact, vielleicht etwas markärmer als normal. Die oberhalb der Vierhügel gelegenen Hirnabschnitte standen mir leider nicht zur Verfügung.

Der mikroskopische Befund an Carminpräparaten zeigt im oberen Ponstheil das Gleiche, wie in der unteren Häfte desselben; nirgends Körnchenzellen; nur um die Gefässe herum gelegentlich mässig starke Anhäufungen von Zellen. Verkalkungen nicht mehr nachweisbar ${ }^{1}$ ).

1) Der eine Brückenschenkel des Kleinhirns wurde noch besonders auf Quierschnitten untersucht, welche nahe dem Pons in senkrechter 
Das $R$ ückenmark liess im obersten Halstheile auch nach der Weigertschen Färbung keine Abnormität erkennen, besonders auch in den Pyramidenbahnen nicht. Im oberen Drittel der Halsanschwellung ist dagegen vor der Pyramidenbahn im rechten Seitenstrange ein kleines, halbstecknadelkopfgrosses Fleckchen zu erkennen. Im mittleren und unteren Drittel der Halsanschwellung tritt eine schwache, aber deutliche, im Dorsaltbeile eine stärkere Degeneration beider Pyramidenbahnen auf, die auch im Lendentheile noch in ausgeprägter Weise vorhanden ist. Die Kleinhirnseitenstrangbahnen, sowie die Türk'schen Stränge sind frei. Von einer Meningitis ist nichts wahrzunehmen; die Gefässe im Wesentlichen obne Veränderung; hie und da verdiekt.

Es ergiebt sich also zunächst in Bezug auf die secandären Degenerationen, welche durch eine primäre Entartung des Kleinhirnes hervorgebracht werden können, dass sowohl Faserbündel, die zu den unteren Oliven gehen, als diese selbst zum Theil entartet vorgefunden wurden. Ebenso wenig kann nach dem, was wir sonst wissen, die Entartung innerhalb der mittleren Kleinhirnstiele und den zugehörigen quer verlaufenden Ponsfasern auffallen; und auch die Degeneration der Bindearme stimmt mit den von Flechsig nach Mangel des Kleinhirns constatirten Befunden überein. Dagegen war keine Degeneration der cerebellaren Rückenmarksbahnen vorhanden, die freilich angesichts der Thatsache, dass diese Faserzüge gewöhnlich nach aufwärts secundär degeneriren, nicht zu erwarten war. Indessen hat ganz neuerdings Marchi (Rivist. sperim. di Freniatr. 1886 XII) (citirt nach dem betreffenden Referate im Neurol. Centralblatt, 1886, S. 559) die Behauptung aufgestellt, dass nach totaler Entfernung des Kleinhirnes bei Hunden und Affen neben der Atrophie der Oliven und der Degeneration aller Kleinhirnstiele auch die Flechsig'sche Kleinhirnseitenstrangbahn mit entarte. Die Bestätigung dieser Behauptung bleibt abzuwarten. Wäre sie richtig, so wäre in unserem Falle bei der ziemlich diffusen, wenn auch

Richtung auf denselben angelegt wurden. Es zeigten sich in demselben zwei central gelegene, nicht mit einander zusammenfliessende Degenerationsheerde, welche den 4. oder 5. Theil der Querschnittsfläche einnehmen und nirgends die Peripherie berühren. Innerbalb dieser Heerde ist die Markscheidensubstanz zum grössten Theile völlig verschwunden; der eine von ihnen erstreckt sich noch auf einen Theil einer grösseren Ganglienzellenanbäufung, dessen von der Degeneration verschont gebliebener Abschnitt seine Fasern behalten hat. 
nicht vollständigen Entartung des Kleinhirns das Fehlen einer auch nur theilweisen Entartung der cerebellaren Rückenmarksbahn neben dem Vorhandensein partieller Degeneration innerhalb aller Kleinhirnstiele doch eine recht auffallende Erscheinung. -

Die Degeneration der Pyramidenbahnen muss natürlich als eine selbständige aufgefasst werden. Es entsteht nur die Frage, ob die innerhalb der Oblongata vorhandene deutliche, wenn auch nicht sehr intensive Entartung der betreffenden Bahnen als die Ursache der im mittleren Halstheil beginnenden und sich nach unten zu verstärkenden Degeneration anzusehen ist oder nicht. Für die gewöhnliche secundäre Degeneration wird allerdings allgemein angenommen, dass sie - wenigstens nach langer Dauer der primären Läsion - sich continuirlich von dem primären Heerde aus nach unten zu fortpflanzt. Für die gegentheilige Annahme, dass bei nachweisbarer primärer Läsion nur der peripherische Theil der zugehörigen, der secundären Degeneration fähigen Bahn selbst bei langer Dauer der primären Affection entarte und der nächstgelegene Abschnitt nicht, oder wenigstens nicht in nachweisbarer Weise, liegen bisher noch keine Beweise vor. Es ist hier um so weniger der Ort, auf diese Frage einzugehen, als bei einem so complicirten Falle wie dem unsrigen sich die Annahme nicht von der Hand weisen lässt, dass dieselbe Ursache, welche die primäre Degenerationen in der Med. obl. verursacht hat, auch direct die - vielleicht durch die Unterbrechung des Ernährungsstromes innerhalb der Medulla oblongata empfänglicher gewordenen - spinalen Pyramidenbahnen geschädigt haben könnte.

Um welchen pathologischen Prozess handelt es sich aber im Kleinhirn und in denjenigen Abschnitten der Med. obl., welche als selbständig und unabhängig von der Kleinhirnerkrankung afficirt angesehen werden müssen? Die bei der multiplen Sklerose im strengen Sinne vorhandene Entartung liegt jedenfalls nicht vor; denr es fehlt die eigenthümliche, gewöhnlich so scharf abschneidende Entartung der Markscheiden in circumscripten Heerden, wie man sie bei der klassischen multiplen Sklerose vorfindet. Es handelt sich in unserem Falle um einen ausgedehnten mehr gleichmässigen Schwund der ganzen Nervenfaser, einen Schwund, welcher allerdings an einzelnen Stellen mehr in Heerdform auftritt. 
Die Veränderungen, welche die Gefässe zeigen, und zwar in der Form von Endarteriitis obliterans, erregen selbstverständlich den Verdacht auf eine syphilitische Erkrankung. Indessen lassen sich eigentliche Granulationsgeschwülste und gummöse Partien nicht finden, wenn auch durchaus zugegeben werden kann, dass einzelne gliareiche Plaques vielleicht früher von starken Rundzellenanhäufungen durchsetzt gewesen sein mögen. Auch die verhältnissmässig geringfügige Betheiligung der Meningen an dem krankhaften Prozess spricht nicht gerade für die syphilitische Natur der vorgefundenen Veränderungen; vor Allem aber wurde in durchaus glaubwürdiger Weise jede luetische Affection bei Lebzeiten des Kranken in Abrede gestellt; und auch in den übrigen Organen liess sich kein syphilitischer Erkrankungsheerd vorfinden, woraus freilich nicht viel geschlossen werden darf. Jedenfalls darf aber wohl die starke, ungleichmässige Verengerung der Gefässe als Ursache für die Entstehung des Nervenfaserschwundes mit herbeigezogen werden, wofür besonders das Vorhandensein des vielen Blutfarbstoffes spricht, welcher sich in der Rinde und in der Marksubstanz des Kleinhirns vorfand und der bekanntlich bei primären, von Gefässveränderungen unabhängigen Degenerationen der Nervenfasern nicht vorgefunden wird. Andrerseits wurde wieder eine andere Erscheinung vermisst, welche nach Gefässerkrankungen in den ischämischen Bezirken aufzutreten pflegt, nehmlich das Vorhandensein von Körnchenzellen. $O b$ die letzteren aber auch bei ganz langsam sich entwickelnden Gefässveränderungen nothwendigerweise auftreten müssen, ist noch vollständig unbekannt. $\mathrm{Zu}$ einem abschliessenden sicheren Urtheile über diese Verhältnisse können wir also zur Zeit noch nicht gelangen, um so weniger, als uns durch die vorhandene Pyramidenbahnerkrankung im Rückenmarke der Gedanke nahe gelegt wird, dass auch neben dem Einfluss der eingesehränkten Blutzufuhr seitens der Gefässe eine directe Schüdigung der betroffenen Nervenfasern durch irgend eine gemeinsame Ursache stattgefunden haben könnte.

Da unser Kranke Jahre lang eine übermässige Quantität von Alkohol genossen hat, so darf man gewiss an den Alkohol als diese Ursache denken. Dass Gefässerkrankungen gleicher Art wie im vorliegenden Falle dureh Einwirkung von Alkohol 
hervorgebracht werden können, wird jedenfalls von Vielen angenommen, während es allerdings fraglich ist, in wie weit chronische Degenerationsprozesse bestimmter Art im Centralnervensysteme durch die gleiche Ursache erzeugt werden. Da der Alkohol ausgebreitete Degenerationen im peripherischen Nervensysteme herbeizuführen im Stande ist, wäre es schwer verständlich, warum er nicht ähnliche Wirkungen auch innerhalb des centralen Nervensýstems zu Wege brächte, auf welches doch seine ersten und vornehmsten Wirkungen gerichtet sind. Es muss sogar auffallend erscheinen, dass nicht das Kleinhirn als der Sitz der Gleichgewichtsfunctionen, die doch so häufig durch den Alkohol beinträchtigt werden, sich öfters erkrankt findet, als es in Wirklichkeit der Fall zu sein scheint. Wären die Pyramidenbahnen besonders durch den Alkohol gefährdet, so würde wohl längst eine Degeneration derselben häufiger bei Potatoren gefunden worden sein. Am meisten leidet nach dem bisher Bekannten wohl noch die Substanz des Grosshirns, die ich leider in unserem Falle nicht untersuchen konnte.

Soviel über die Deutung des anatomischen Befundes und die wahrscheinliche Ursache der Erkrankang. Von den beobachteten klinischen Erscheinungen lässt sich die eigenthümliche Gehstörung auf die Erkrankung des Kleinhirnes zurückführen, während die Sprachstörung wohl auf die unzweifelhaft vorhandene Degeneration des Fasernetzes innerhalb der Hypoglossuskerne und der von diesen centralwärts ziehenden Fasern zu beziehen ist. Da der Diabetes insipidus schon vor der Erkrankung des Kleinhirnes, des Pons und der Med. obl. bestanden hat, so lässt sich natürlich irgend eine Vermuthung über seine Entstehungsweise nicht geben. Ebensowenig lässt sich sagen, wodurch speciell der leichte Nystagmus und das leichte Intentionszittern der Arme herbeigeführt wurde. Da die gewöhnliche absteigende Degeneration der Pyramidenbahn allein die letztgenannte Erscheinung nicht herbeizuführen pflegt, so wird es wahrscheinlich gemacht, dass dieses Zittern auch durch Erkrankungen des Pons und der Med. obl. erzeugt werden kann; isolirte cerebellare Erkrankung ziehen es nach unseren bisherigen Erfahrungen ebenfalls nicht nach sich. 